\title{
Comparison between the Laplace Decomposition Method and Adomian Decomposition in Time-Space Fractional Nonlinear Fractional Differential Equations
}

\author{
Mohamed Z. Mohamed1, Tarig M. Elzaki2* \\ ${ }^{1}$ Mathematics Department, Faculty of Sciences, Sudan University of Science and Technology, Khartoum State, Sudan \\ ${ }^{2}$ Mathematics Department, Faculty of Sciences and Arts-Alkamil, University of Jeddah, Jeddah, Saudi Arabia \\ Email: Lesha-83@hotmail.com, *Tarig.alzaki@gmail.com
}

How to cite this paper: Mohamed, M.Z. and Elzaki, T.M. (2018) Comparison between the Laplace Decomposition Method and Adomian Decomposition in Time-Space Fractional Nonlinear Fractional Differential Equations. Applied Mathematics, 9, 448-458. https://doi.org/10.4236/am.2018.94032

Received: March 15, 2018

Accepted: April 27, 2018

Published: April 30, 2018

Copyright ( 2018 by authors and Scientific Research Publishing Inc. This work is licensed under the Creative Commons Attribution International License (CC BY 4.0).

http://creativecommons.org/licenses/by/4.0/

\begin{abstract}
The aim of this paper is to discuss application of Laplace Decomposition Method with Adomian Decomposition in time-space Fractional Nonlinear Fractional Differential Equations. The approximate solutions result from Laplace Decomposition Method and Adomian decomposition; those two accessions are comfortable to perform and firm when to PDEs. For caption and further representation of the thought, several examples are tool up.
\end{abstract}

\section{Keywords}

Laplace Decomposition Method, Mittag-Leffler Function, Partial Fractional Differential Equation

\section{Introduction}

Recently, research has shown that numerous phenomena in fluid mechanics, viscoelasticity, biology, physics, engineering and other field from knowledge mastery are successfully modeled by the use of FPDEs.

Researchers developed numerous methods to resolve FODEs, integral equations and fractional partial differential equations of physical interest. The flowing is an illustration of several of the generality used ones; ADM [3] [4] [5] [6], VIM [7], FDM [2], DTM [9], HPM [8]. Among the already mentioned ones, the decomposition method stood up as efficient, easy and accurate in solving a great group of linear and nonlinear ordinary, partial, deterministic or stochastic differential equations. This method is fully appropriate to physically solve problems, some time ago it did not request superfluous linearization, and other bound methods and postulate whom may vary the problem being solved [10] [11]. The 
LDM is a numerical algorithm to solve NODEs and PDEs. Khuri [13] [14] developed this method for the sacrificial solution of a class of NODEs. These numerical technique styles ultimately clarify how the Laplace Transform supposedly is utilized to sacrifice the solutions of the nonlinear differential equations by impacting the Decomposition Method that was initially before Adomian.

\section{The Definitions of Fractional Calculus and Laplace Transform}

Definition 2.1 Aral function $f(t), t>0$ is said to be the space $C_{\mu}, \mu \in \mathbb{R}$, if the there exists a real number $p>\mu$ such that $f(t)=t^{p} f_{1}(t)$, where $f_{1}(t) \in C[0, \infty)$, clearly $C_{\mu} \subset C_{\beta}$ if $\beta \leq \mu$.

Definition 2.2 The left sides Riemann-Liouville fractional integral operator of order $v \geq 0$ of a function $f(t) \in C_{\mu}, \mu \geq-1$ is defined as [1]-[15]

$$
J^{v} f(t)= \begin{cases}\frac{1}{\Gamma(v)} \int_{0}^{t}(t-\xi)^{v-1} f(\xi) \mathrm{d} \xi & v, t>0, \\ f(t) & v=0 .\end{cases}
$$

Definition 2.3 The left sided Caputo fractional derivative of $f$, $f \in C_{-1}^{m}, m \in \mathbb{N} \cup\{0\}$ is defined as [1]-[15]

$$
D^{v} f(t)=\frac{\partial^{v} f(t)}{\partial t^{v}}=\left\{\begin{array}{lc}
J^{m-v}\left[\frac{\partial^{m} f(t)}{\partial t^{m}}\right], & m-1<v \leq m, m \in \mathbb{N}, \\
\frac{\partial^{m} f(t)}{\partial t^{m}} & v=m .
\end{array}\right.
$$

Hence, we have the following properties [1]-[15]

$$
\begin{aligned}
& \text { 1. } J^{v} J^{\alpha} f(t)=J^{v+\alpha} f(t), \quad \alpha, v \geq 0 . \\
& \text { 2. } J^{v} t^{\gamma}=\frac{\Gamma(\gamma+1)}{\Gamma(v+\gamma+1)} t^{v+\gamma}, \alpha>0, \gamma>-1, t>0 . \\
& \text { 3. } J^{v} D_{*}^{v} f(t)=f(t)-\sum_{k=0}^{m-1} f^{(k)}\left(0^{+}\right) \frac{t^{k}}{k !}, t>0, m-1<v \leq m
\end{aligned}
$$

Definition 2.4 If $m-1<v \leq m, m \in \mathbb{N}$, then the Laplace transform of the fractional derivative $D_{*}^{v} f(t)$ is

$$
L\left[D_{*}^{v} f(t)\right]=s^{v} F(s)-\sum_{k=0}^{m-1} f^{(k)}\left(0^{+}\right) s^{v-k-1}, t>0,
$$

where $F(s)$ be the Laplace transform of $f(t)$ [1] [2] [3].

Definition 2.5 The Mittag-Leffler function $E_{v}(t)$ with $v>0$ is defined by the following series representation, valid in the whole complex plane [2]

$$
E_{v}(t)=\sum_{k=0}^{\infty} \frac{t^{k}}{\Gamma(k v+1)}
$$

\section{The Main Idea of Fractional Laplace Decomposition Method}

In this section the class of Nonlinear Fractional Differential Equation is consi- 
dered.

$$
D^{v} u(x, t)+R u(x, t)+N u(x, t)=f(x, t), \quad x, t \geq 0, m-1<v<m .
$$

where $D^{v}=\frac{\partial^{v}}{\partial t^{v}}$ the Caputo fractional derivative of is order $v, m \in \mathrm{N}$, where $R$ is a linear operator, $N$ is a nonlinear function and $f$ is the source function. The initial and boundary associated with Equation (3.1) are of the from

$$
u(x, 0)=h(x), \quad 0<v \leq 1, \quad t>0,
$$

And

$$
\frac{\partial u(x, 0)}{\partial t}=k(x), 1<v \leq 2, t>0
$$

According to the Applying of the Laplace transform to Equation (3.1) and the use of linearity Laplace transform, the result is

$$
L\left[D^{v} u(x, t)\right]+L[R u(x, t)+N u(x, t)]=L[f(x, t)],
$$

when the property of Laplace transform is used, we get

$$
\begin{gathered}
s^{v} u(x, s)-s^{v-1} u(x, 0)-s^{v-2} u_{t}(x, 0)=L[f(x, t)]-L[R u(x, t)+N u(x, t)], \\
u(x, s)=\frac{h(x)}{s}+\frac{k(x)}{s^{2}}+\frac{L}{s^{v}}[f(x, t)]-\frac{L}{s^{v}}[R u(x, t)+N u(x, t)],
\end{gathered}
$$

Stander Laplace decomposition defines the solution $u(x, t)$ before the series

$$
u(x, t)=\sum_{n=0}^{\infty} u_{n}(x, t)
$$

The Nonlinear operator is decomposed as follows:

$$
N u(x, t)=\sum_{n=0}^{\infty} A_{n}
$$

See $A_{n}$ the Adomian polynomial that are given by

$$
A_{n}=\frac{1}{n !} \frac{\mathrm{d}^{n}}{\mathrm{~d} \lambda^{n}}\left[N\left(\sum_{i=0}^{\infty} \lambda^{i} u_{i}\right)\right]_{\lambda=0}, n=1,2, \cdots .
$$

Substitution Equation (3.7), Equation (3.8) and Equation (3.9) to Equation (3.6), we have

$$
\sum_{n=0}^{\infty} u_{n}(x, s)=\frac{h(x)}{s}+\frac{k(x)}{s^{2}}+\frac{L}{s^{v}}[f(x, t)]-\frac{L}{s^{v}}\left[R \sum_{n=0}^{\infty} u_{n}(x, t)+\sum_{n=0}^{\infty} A_{n}\right],
$$

when both sides of Equation (3.10) are matched, the following iterative algorithm is yielded:

$$
\begin{gathered}
u_{0}(x, s)=\frac{h(x)}{s}+\frac{k(x)}{s^{2}}+\frac{L}{s^{v}}[f(x, t)]=g(x, s) \\
u_{1}(x, s)=-\frac{L}{s^{v}}\left[R u_{0}(x, t)+A_{0}\right], u_{2}(x, s)=-\frac{L}{s^{v}}\left[R u_{1}(x, t)+A_{1}\right]
\end{gathered}
$$

Generally, the recursive relation is given as follows 


$$
u_{n+1}(x, s)=-\frac{L}{s^{v}}\left[R u_{n}(x, t)+A_{n}\right], \quad n \geq 1
$$

When the inverse Laplace transform Equation (3.13) is applied, the following is obtained

$$
\begin{gathered}
u_{0}(x, t)=g(x, t) \\
u_{n+1}(x, t)=-L^{-1}\left[\frac{L}{s^{v}}\left[R u_{n}(x, t)+A_{n}\right]\right], n \geq 1
\end{gathered}
$$

where $g(x, t)$ is a function that arises from the source term and the prescribed initial condition, the initial solution is important, the choice of Equation (3.15) as the initial solution always leads to noise oscillation during the iteration procedure.

\section{Numerical Results}

To show the method of coupled fractional nonlinear partial differential equations, three examples are considered in this section.

Example 1 Consider the nonlinear time-fractional advection partial differential equation [11].

$$
D^{v} u_{t}(x, t)+u(x, t) u_{x}(x, t)=x+x t^{2}, t>0,0<v \leq 1,
$$

The initial condition is subject to:

$$
u(x, t)=0
$$

When the Laplace transform is applied to both sides of Equation (4.1) the following is yielded.

$$
s^{v} u(x, s)=s^{v-1} u(x, 0)+L\left[x+x t^{2}\right]+L\left[u(x, t) u_{x}(x, t)\right]
$$

By using the initial condition, the following recurrence relational is yielded

$$
u(x, s)=\frac{x}{s^{v+1}}+\frac{2 x}{s^{v+3}}-\frac{1}{s^{v}} L\left[u(x, t) u_{x}(x, t)\right]
$$

Applying the inverse Laplace transform, to both sides of Equation (4.4) yields:

$$
u(x, t)=\frac{x}{\Gamma(v+1)} t^{v}+\frac{2 x}{\Gamma(v+3)} t^{v+2}-L^{-1}\left[\frac{1}{s^{v}} L\left[u(x, t) u_{x}(x, t)\right]\right]
$$

The LDM proposes a series solution of the function $u(x, t)$ which is given series Equation (3.7) and using Equation (3.7) into Equation (4.5)

$$
\sum_{n=0}^{\infty} u_{n}(x, t)=\frac{x}{\Gamma(v+1)} t^{v}+\frac{2 x}{\Gamma(v+3)} t^{v+2}-L^{-1}\left[\frac{1}{s^{v}} L\left[\sum_{n=0}^{\infty} A_{n}\right]\right]
$$

In above Equation(4.6) is Adomian polynomials that represents nonlinear terms. The few components of Adomian polynomials

$$
\begin{aligned}
& A_{0}=u_{0} u_{0 x} \\
& A_{1}=u_{0 x} u_{1}+u_{0} u_{1 x} \\
& A_{2}=u_{0 x} u_{2}+u_{1 x} u_{1}+u_{2 x} u_{0} \\
& A_{3}=u_{0 x} u_{3}+u_{1 x} u_{2}+u_{2 x} u_{1}+u_{3 x} u_{0}
\end{aligned}
$$


Then following recurrence relations, we have

$$
\begin{gathered}
u_{0}(x, t)=\frac{x}{\Gamma(v+1)} t^{v}+\frac{2 x}{\Gamma(v+3)} t^{v+2} \\
u_{n+1}(x, t)=-L^{-1}\left[\frac{1}{s^{v}} L\left[\sum_{n=0}^{\infty} A_{n}\right]\right]
\end{gathered}
$$

Consequently

$$
\begin{aligned}
& u_{1}(x, t)=-L^{-1}\left[\frac{1}{s^{v}} L\left[\sum_{n=0}^{\infty} A_{0}\right]\right] \\
& =-\frac{x \Gamma(2 v+1)}{\Gamma^{2}(v+1) \Gamma(3 v+1)} t^{3 v}-\frac{4 x \Gamma(2 v+3)}{\Gamma(v+1) \Gamma(v+3) \Gamma(3 v+3)} t^{3 v+2} \\
& \quad-\frac{4 x \Gamma(2 v+5)}{\Gamma^{2}(v+3) \Gamma(3 v+5)} t^{3 v+4} \\
& u_{2}(x, t)=-L^{-1}\left[\frac{1}{s^{v}} L\left[\sum_{n=0}^{\infty} A_{1}\right]\right] \\
& =\frac{2 x \Gamma(2 v+1) \Gamma(4 v+1)}{\Gamma^{3}(v+1) \Gamma(3 v+1) \Gamma(5 v+1)} t^{5 v} \\
& +\left[\frac{8 x \Gamma(2 v+3)}{\Gamma^{2}(v+1) \Gamma(v+3) \Gamma(3 v+3)}+\frac{4 x \Gamma(2 v+3)}{\Gamma(v+1) \Gamma^{2}(v+3) \Gamma(3 v+3)}\right] \frac{\Gamma(4 v+3)}{\Gamma(5 v+3)} t^{5 v+2} \\
& +\left[\frac{8 x \Gamma(2 v+5)}{\Gamma(v+1) \Gamma^{2}(v+3) \Gamma(3 v+5)}+\frac{16 x \Gamma(2 v+3)}{\Gamma(v+1) \Gamma^{2}(v+3) \Gamma(3 v+3)}\right] \frac{\Gamma(4 v+5)}{\Gamma(5 v+5)} t^{5 v+4} \\
& +\frac{16 x \Gamma(2 v+5) \Gamma(4 v+7)}{\Gamma^{3}(v+3) \Gamma(3 v+5) \Gamma(5 v+7)} t^{5 v+6}
\end{aligned}
$$

The third term approximating a solution to Equation (4.1) gives the following:

$$
\begin{aligned}
u(x, t)= & \frac{x}{\Gamma(v+1)} t^{v}+\frac{2 x}{\Gamma(v+3)} t^{v+2}+\frac{x \Gamma(2 v+1)}{\left.\Gamma^{2}(v+1) \Gamma(3 v+1)\right)} t^{3 v} \\
& -\frac{4 x \Gamma(2 v+3)}{\Gamma(v+1) \Gamma(v+3) \Gamma(3 v+3)} t^{3 v+2} \\
& -\frac{4 x \Gamma(2 v+5)}{\Gamma^{2}(v+3) \Gamma(3 v+5)} t^{3 v+4}+\cdots
\end{aligned}
$$

Example 4.2 Consider the nonlinear time-fractional hyperbolic equation [16].

$$
D^{v} u_{t}(x, t)=\frac{\partial}{\partial x}\left[u(x, t) u_{x}(x, t)\right], t>0,1<v \leq 2
$$

The initial condition is subject to:

$$
u(x, t)=x^{2}, u_{t}(x, t)=-2 x^{2}
$$

When the Laplace transform is applied to both sides of Equation (4.8) the following is yielded. 


$$
s^{v} u(x, s)=s^{\nu-1} u(x, 0)+s^{v-2} u_{t}(x, 0)+L\left[\frac{\partial}{\partial x}\left[u(x, t) u_{x}(x, t)\right]\right]
$$

By using initial conditions, the following recurrence relational is found

$$
u(x, s)=\frac{x^{2}}{s}-\frac{2 x^{2}}{s^{2}}+\frac{L}{s^{v}}\left[\frac{\partial}{\partial x}\left[u(x, t) u_{x}(x, t)\right]\right]
$$

When the inverse Laplace transform, is applied, to both sides of Equation (4.11) the following is yielded:

$$
u(x, t)=x^{2}-2 x^{2} t^{2}+L^{-1}\left[\frac{L}{s^{v}}\left[\frac{\partial}{\partial x}\left[u(x, t) u_{x}(x, t)\right]\right]\right]
$$

The LDM the LDM proposes a series solution of the function $u(x, t)$ which is given series Equation (3.6) and using Equation (3.6) into Equation (4.12)

$$
\sum_{n=0}^{\infty} u_{n}(x, t)=x^{2}-2 x^{2} t^{2}+L^{-1}\left[\frac{L}{s^{v}}\left[\frac{\partial}{\partial x}\left[\sum_{n=0}^{\infty} A_{n}\right]\right]\right]
$$

In above Equation (4.13) Adomian polynomials represent nonlinear terms $N=u u_{x}$. The few components of the decomposition series are derived as follows:

$$
\begin{gathered}
u_{0}(x, t)=x^{2}-2 x^{2} t \\
u_{1}(x, t)=6 x^{2}\left(\frac{t^{v}}{\Gamma(v+1)}-\frac{4 t^{v+1}}{\Gamma(v+2)}+\frac{8 t^{v+2}}{\Gamma(v+3)}\right) \\
u_{2}(x, t)=72 x^{2}\left(\frac{t^{2 v}}{\Gamma(3 v+1)}-4 \frac{t^{2 v+1}}{\Gamma(2 v+2)}\right. \\
\left.+2 \frac{\Gamma(v+1) t^{2 v+1}}{\Gamma(2 v+4) \Gamma(v+1)}-16 \frac{\Gamma(v+4) t^{2 v+3}}{\Gamma(2 v+4) \Gamma(v+3)}\right) \\
+72 x^{2}\left[8 \frac{1}{\Gamma(2 v+3)}+8 \frac{\Gamma(v+3)}{\Gamma(2 v+3) \Gamma(v+2)}\right] t^{2 v+2}
\end{gathered}
$$

\section{Example 4.3}

Consider a system of nonlinear coupled with partial differential equation [16].

$$
\begin{gathered}
D^{v} u_{t}(x, y, t)+v_{x} w_{y}-v_{y} w_{x}=-u \\
D^{v} v_{t}(x, y, t)+w_{x} u_{y}+u_{x} w_{y}=v \\
D^{v} w_{t}(x, y, t)+u_{x} v_{y}+u_{y} v_{x}=w
\end{gathered}
$$

The initial condition is subject to:

$$
\begin{gathered}
u(x, y, 0)=\mathrm{e}^{x+y} \\
v(x, y, 0)=\mathrm{e}^{x-y} \\
w(x, y, 0)=\mathrm{e}^{-x+y}
\end{gathered}
$$

When the Laplace transform is applied the both sides of [Equation (4.14), Equation (4.15) and Equation (4.16)] through using the initial condition, the fol- 
lowing recurrence relational is yielded

$$
\begin{gathered}
u(x, y, s)=\frac{\mathrm{e}^{x+y}}{s}-\frac{L}{s^{\alpha}}\left[v_{x} w_{y}-v_{y} w_{x}-u\right] \\
v(x, y, s)=\frac{\mathrm{e}^{x-y}}{s}+\frac{L}{s^{\beta}}\left[v-w_{x} u_{y}-u_{x} w_{y}\right] \\
w(x, y, s)=\frac{\mathrm{e}^{-x+y}}{s}+\frac{L}{s^{\mu}}\left[w-u_{x} v_{y}-u_{y} v_{x}\right]
\end{gathered}
$$

Through applying inverse Laplace transform to both sides of [Equation (4.20), Equation (4.21) and Equation (4.22)] we can yields.

$$
\begin{aligned}
& u(x, y, t)=\mathrm{e}^{x+y}-L^{-1}\left[\frac{L}{s^{\alpha}}\left[v_{x} w_{y}-v_{y} w_{x}-u\right]\right] \\
& v(x, y, t)=\mathrm{e}^{x-y}+L^{-1}\left[\frac{L}{s^{\beta}}\left[v-w_{x} u_{y}-u_{x} w_{y}\right]\right] \\
& w(x, y, t)=\mathrm{e}^{-x+y}+L^{-1}\left[\frac{L}{s^{\mu}}\left[w-u_{x} v_{y}-u_{y} v_{x}\right]\right]
\end{aligned}
$$

The LADM assumes a series solution of the function $u(x, t)$ which is given series Equation (3.6) and through using Equation (3.6) into [Equation (4.23), Equation (4.24) and Equation (4.25)] yielding.

$$
\begin{gathered}
\sum_{n=0}^{\infty} u_{n}(x, y, t)=\mathrm{e}^{x+y}-L^{-1}\left[\frac{L}{s^{\alpha}}\left[\sum_{n=0}^{\infty} F_{n}(v, w)-\sum_{n=0}^{\infty} G_{n}(v, w)-u_{n}\right]\right] \\
\sum_{n=0}^{\infty} v_{n}(x, y, t)=\mathrm{e}^{x-y}+L^{-1}\left[\frac{L}{s^{\beta}}\left[v_{n}-\sum_{n=0}^{\infty} H_{n}(u, w)-\sum_{n=0}^{\infty} I_{n}(u, w)\right]\right] \\
\sum_{n=0}^{\infty} w_{n}(x, y, t)=\mathrm{e}^{-x+y}+L^{-1}\left[\frac{L}{s^{\mu}}\left[w_{n}-\sum_{n=0}^{\infty} J_{n}(u, v)-\sum_{n=0}^{\infty} K_{n}(u, v)\right]\right]
\end{gathered}
$$

where $F_{n}(v, w), G_{n}(v, w), H_{n}(v, w), I_{n}(v, w), J_{n}(v, w)$ and $K_{n}(v, w)$ are Adomian polynomials they represent nonlinearities arising in above system [Equation (4.26), Equation (4.27) and Equation (4.28)] of nonlinear coupled partial differential equations. The components of above Adomian polynomials are given below

$$
\begin{aligned}
F_{0}(v, w) & =v_{0 y} w_{0 x} \\
F_{1}(v, w) & =v_{1 y} w_{0 x}+v_{0 y} w_{1 x} \\
F_{2}(v, w) & =v_{0 y} w_{2 x}+v_{1 y} w_{1 x}+v_{2 y} w_{0 x} \\
\vdots & \\
F_{n}(v, w)= & \sum_{i=0}^{\infty} v_{i y} w_{n-1 x} \\
G_{0}(v, w) & =v_{0 x} w_{0 y} \\
G_{1}(v, w) & =v_{1 x} w_{0 y}+v_{0 x} w_{1 y} \\
G_{2}(v, w) & =v_{0 x} w_{2 y}+v_{1 x} w_{1 y}+v_{2 x} w_{0 y} \\
& \vdots \\
G_{n}(v, w) & =\sum_{i=0}^{\infty} v_{i x} w_{n-1 y}
\end{aligned}
$$




$$
\begin{aligned}
H_{0}(v, w) & =w_{0 x} u_{0 y} \\
H_{1}(v, w) & =w_{1 x} u_{0 y}+w_{0 x} u_{1 y} \\
H_{2}(v, w) & =w_{0 x} u_{2 y}+w_{1 x} u_{1 y}+w_{2 x} u_{0 y} \\
& \vdots \\
H_{n}(v, w) & =\sum_{i=0}^{\infty} w_{i x} u_{n-1 y} \\
I_{0}(v, w) & =w_{0 y} u_{0 x} \\
I_{1}(v, w) & =w_{1 y} u_{0 x}+w_{0 y} u_{1 x} \\
I_{2}(v, w) & =w_{0 y} u_{2 x}+w_{1 y} u_{1 x}+w_{2 y} u_{0 x} \\
& \vdots \\
I_{n}(v, w)= & \sum_{i=0}^{\infty} w_{i y} u_{n-1 x} \\
J_{0}(v, w)= & u_{0 x} v_{0 y} \\
J_{1}(v, w)= & u_{1 x} v_{0 y}+u_{0 x} v_{1 y} \\
J_{2}(v, w)= & u_{0 x} v_{2 y}+u_{1 x} v_{1 y}+u_{2 x} v_{0 y} \\
\vdots & \\
J_{n}(v, w) & =\sum_{i=0}^{\infty} u_{i x} v_{n-1 y} \\
K_{0}(v, w) & =u_{0 y} v_{0 x} \\
K_{1}(v, w) & =u_{1 y} v_{0 x}+u_{0 y} v_{1 x} \\
K_{2}(v, w) & =u_{0 y} v_{2 x}+u_{1 y} v_{1 x}+u_{2 y} v_{0 x} \\
& \vdots \\
K_{n}(v, w) & =\sum_{i=0}^{\infty} u_{i y} v_{n-1 x}
\end{aligned}
$$

The following recursive relation is obtained:

$$
\begin{gathered}
u_{0}(x, y, t)=\mathrm{e}^{x+y}, u_{n+1}(x, y, t) \\
=L^{-1}\left[\frac{L}{s^{\alpha}}\left[\sum_{n=0}^{\infty} F_{n}(v, w)-\sum_{n=0}^{\infty} G_{n}(v, w)-u_{n}\right]\right] \\
v_{0}(x, y, t)=\mathrm{e}^{x-y}, v_{n+1}(x, y, t) \\
=L^{-1}\left[\frac{L}{s^{\beta}}\left[v_{n}-\sum_{n=0}^{\infty} H_{n}(u, w)-\sum_{n=0}^{\infty} I_{n}(u, w)\right]\right] \\
w_{0}(x, y, t)=\mathrm{e}^{-x+y}, w_{n+1}(x, y, t) L^{-1}\left[\frac{L}{s^{\mu}}\left[w_{n}-\sum_{n=0}^{\infty} J_{n}(u, v)-\sum_{n=0}^{\infty} K_{n}(u, v)\right]\right] \\
u_{1}(x, y, t)=L^{-1}\left[\frac{L}{s^{\alpha}}\left[F_{0}(v, w)-G_{0}(v, w)-u_{0}\right]\right] \\
=L^{-1}\left[\frac{L}{s^{\alpha}}\left[v_{0 y} w_{0 x}-v_{0 x} w_{0 y}-u_{0}\right]\right] \\
=L^{-1}\left[\frac{L}{s^{\alpha}}\left[-\mathrm{e}^{x+y}\right]\right]=-\frac{\mathrm{e}^{x+y}}{\Gamma(v+1)} t^{v}
\end{gathered}
$$




$$
\begin{aligned}
& v_{1}(x, y, t)= L^{-1}\left[\frac{L}{s^{\beta}}\left[v_{0}-H_{0}(u, w)-I_{0}(u, w)\right]\right] \\
&= L^{-1}\left[\frac{L}{s^{\beta}}\left[\mathrm{e}^{x-y}-u_{0 y} w_{0 x}-u_{0 x} w_{0 y}\right]\right]=L^{-1}\left[\frac{L}{s^{\beta}}\left[\mathrm{e}^{x-y}\right]\right]=\frac{\mathrm{e}^{x-y}}{\Gamma(\beta+1)} t^{\beta} \\
& w_{1}(x, y, t)=L^{-1}\left[\frac{L}{s^{\mu}}\left[w_{0}-J_{0}(u, v)-K_{0}(u, v)\right]\right] \\
&=L^{-1}\left[\frac{L}{s^{\mu}}\left[\mathrm{e}^{-x+y}-u_{0 x} v_{0 y}-u_{0 y} v_{0 x}\right]\right]=L^{-1}\left[\frac{L}{s^{\mu}}\left[\mathrm{e}^{-x+y}\right]\right]=\frac{\mathrm{e}^{-x+y}}{\Gamma(\beta+1)} t^{\beta} \\
& u_{2}(x, y, t)=L^{-1}\left[\frac{L}{s^{\alpha}}\left[F_{1}(v, w)-G_{1}(v, w)-u_{1}\right]\right] \\
&=L^{-1}\left[\frac{L}{s^{\alpha}}\left[\frac{\mathrm{e}^{x+y}}{\Gamma(\alpha+1)} t^{\alpha}\right]\right]=\frac{\mathrm{e}^{x+y}}{\Gamma(2 \alpha+1)} t^{2 \alpha} \\
& v_{2}(x, y, t)=L^{-1}\left[\frac{L}{s^{\beta}}\left[v_{2}-H_{2}(u, w)-I_{2}(u, w)\right]\right] \\
& w_{2}(x, y, t)=L^{-1}\left[\frac{L}{s^{\mu}}\left[w_{1}-J_{1}(u, v)-K_{1}(u, v)\right]\right. \\
&\left.=L^{-1}\left[\frac{L}{s^{\beta}}\left[\frac{\mathrm{e}^{x-y}}{\Gamma(\beta+y} t^{\mu}\right]\right]=\frac{\mathrm{e}^{-x+y}}{\Gamma(2 \beta+1)} t^{\beta}\right] t^{2 \beta}
\end{aligned}
$$

So the expected solutions are as follows:

$$
\begin{aligned}
u(x, y, t) & =\sum_{n=0}^{\infty} u_{n}(x, y, t) \\
& =\mathrm{e}^{x+y}+\frac{\mathrm{e}^{x+y}}{\Gamma(\alpha+1)} t^{\alpha}+\frac{\mathrm{e}^{x+y}}{\Gamma(2 \alpha+1)} t^{2 \alpha}+\cdots \\
& =\mathrm{e}^{x+y}\left[1+\frac{t^{\alpha}}{\Gamma(\alpha+1)}+\frac{t^{2 \alpha}}{\Gamma(2 \alpha+1)}+\cdots\right] \\
& =\mathrm{e}^{x+y} \sum_{k=0}^{\infty} \frac{t^{k \alpha}}{\Gamma(k \alpha+1)}
\end{aligned}
$$

The solution in a closed form is given as

$$
\begin{aligned}
& =\mathrm{e}^{x+y} E_{\alpha, 1}\left(t^{\alpha}\right) \\
v(x, y, t)= & \sum_{n=0}^{\infty} v_{n}(x, y, t) \\
= & \mathrm{e}^{x-y}+\frac{\mathrm{e}^{x-y}}{\Gamma(\beta+1)} t^{\beta}+\frac{\mathrm{e}^{x-y}}{\Gamma(2 \beta+1)} t^{2 \beta}+\cdots \\
= & \mathrm{e}^{x-y}\left[1+\frac{t^{\beta}}{\Gamma(\beta+1)}+\frac{t^{2 \beta}}{\Gamma(2 \beta+1)}+\cdots\right] \\
= & \mathrm{e}^{x-y} \sum_{k=0}^{\infty} \frac{t^{k \beta}}{\Gamma(k \beta+1)}
\end{aligned}
$$


The solution in a closed form is given as

$$
\begin{aligned}
& =\mathrm{e}^{x-y} E_{\beta, 1}\left(t^{\beta}\right) \\
w(x, y, t) & =\sum_{n=0}^{\infty} w_{n}(x, y, t) \\
& =\mathrm{e}^{-x+y}+\frac{\mathrm{e}^{-x+y}}{\Gamma(\mu+1)} t^{\mu}+\frac{\mathrm{e}^{-x+y}}{\Gamma(2 \mu+1)} t^{2 \mu}+\cdots \\
& =\mathrm{e}^{-x+y}\left[1+\frac{t^{\mu}}{\Gamma(\mu+1)}+\frac{t^{2 \mu}}{\Gamma(2 \mu+1)}+\cdots\right] \\
= & \mathrm{e}^{-x+y} \sum_{k=0}^{\infty} \frac{t^{k \mu}}{\Gamma(k \mu+1)}
\end{aligned}
$$

The solution in a closed structure is given as

$$
=\mathrm{e}^{-x+y} E_{\mu, 1}\left(t^{\mu}\right)
$$

\begin{tabular}{|c|c|c|c|c|c|c|c|c|}
\hline \multirow{2}{*}{$t$} & \multirow{2}{*}{$x$} & \multicolumn{2}{|c|}{$v=0.5$} & \multicolumn{2}{|c|}{$v=0.75$} & \multicolumn{2}{|c|}{$v=1$} & \multirow{2}{*}{ exact } \\
\hline & & $L D M$ & $A D M$ & $L D M$ & $A D M$ & $L D M$ & $A D M$ & \\
\hline \multirow{4}{*}{0.2} & 0.2500 & 0.11276 & 0.112844 & 0.07878 & 0.078787 & 0.050000 & 0.050000 & 0.05000 \\
\hline & 0.5000 & 0.22553 & 0.225688 & 0.15756 & 0.157574 & 0.100000 & 0.100000 & 0.10000 \\
\hline & 0.7500 & 0.33829 & 0.311249 & 0.23635 & 0.236361 & 0.150000 & 0.150001 & 0.15000 \\
\hline & 1.0000 & 0.45106 & 0.451375 & 0.31513 & 0.315148 & 0.200000 & 0.200001 & 0.20000 \\
\hline \multirow{4}{*}{0.4} & 0.2500 & 0.162261 & 0.164004 & 0.128775 & 0.128941 & 0.100011 & 0.100023 & 0.10000 \\
\hline & 0.5000 & 0.324523 & 0.328008 & 0.257550 & 0.128941 & 0.200022 & 0.200046 & 0.20000 \\
\hline & 0.7500 & 0.486785 & 0.492011 & 0.386326 & 0.386821 & 0.300033 & 0.300069 & 0.30000 \\
\hline & 1.0000 & 0.649047 & 0.656015 & 0.515101 & 0.515762 & 0.400045 & 0.400092 & 0.40000 \\
\hline
\end{tabular}

\section{Conclusion}

In this treatise, Laplace decomposition method has been successfully used to solve the approximate solution of nonlinear PFDEs (see Table 1 and Table 2).

Table 1. Numerical values for Equation (4.1) [11].

Table 2. Numerical values for Equation (4.8) [16].

\begin{tabular}{cccccc}
\hline \multirow{2}{*}{$t$} & & $v=0.5$ & $v=0.75$ & $v=1$ & \multirow{2}{*}{ exact } \\
\cline { 3 - 5 } & & \multicolumn{1}{c}{$L D M$} & $L D M$ & $L D M$ & \\
\hline \multirow{2}{*}{0.2} & 0.25 & 0.059283 & 0.048701 & 0.043395 & 0.043403 \\
& 0.50 & 0.237132 & 0.194804 & 0.173580 & 0.173611 \\
& 0.75 & 0.533549 & 0.438311 & 0.390555 & 0.390625 \\
& 1.0 & 0.948531 & 0.779219 & 0.694321 & 0.694445 \\
& 0.25 & 0.065411 & 0.043748 & 0.031566 & 0.031887 \\
& 0.50 & 0.261647 & 0.174992 & 0.126267 & 0.127551 \\
& 0.75 & 0.588706 & 0.393732 & 0.284102 & 0.286989 \\
& 1.0 & 1.046589 & 0.699968 & 0.505071 & 0.510204 \\
\hline
\end{tabular}


The solutions of these examples of methods are utilized for the solution of high-order initial value problems.

\section{References}

[1] Miller, K.S. and Ross, B. (1993) An Introduction to the Fractional Calculus and Fractional Differential Equations. John Wiley and Sons, New York.

[2] Podlubny, I. (1999) Fractional Differential Equations. Academic Press, San Diego, CA.

[3] Song, L. and Wang, W. (2013) A New Improved Adomian Decomposition Method and Its Application to Fractional Differential Equations. Applied Mathematical Modelling, 37, 1590-1598. https://doi.org/10.1016/j.apm.2012.03.016

[4] Herzallah, M.A.E. and Gepreel, K.A. (2012) Approximate Solution to the Time-Space Fractional Cubic Nonlinear Schrodinger Equation. Applied Mathematical Modelling, 36, 5678-5685. https://doi.org/10.1016/j.apm.2012.01.012

[5] Faraz, N., Khan, Y. and Sankar, D.S. (2010) Decomposition-Transform Method for Fractional Differential Equations. International Journal of Nonlinear Sciences and Numerical Simulation, 11, 305-310.

[6] Faraz, N., Khan, Y., Jafari, H., Yildirim, A. and Madani, M. (2011) Fractional Variational Iteration Method via Modified Riemann-Liouville Derivative. Journal of King Saud University—Science, 23, 413-417. https://doi.org/10.1016/j.jksus.2010.07.025

[7] Faraz, N. (2011) Study of the Effects of the Reynolds Number on Circul Arborous Slider via Variational Iteration Algorithm-II. Computers \& Mathematics with Applications, 61, 1991-1994. https://doi.org/10.1016/j.camwa.2010.08.048

[8] Kumar, S. and Singh, O.P. (2010) Numerical Inversion of the Abel integral Equation Using Homotopy Perturbation Method. Z Naturforschung, 65a, 677-682.

[9] Rashidi, M.M. (2009) The Modified Differential Transform Method for Solving MHD Boundary-Layer Equations. Computer Physics Communications, 180, 2210-2217. https://doi.org/10.1016/j.cpc.2009.06.029

[10] Wazwaz, A.M. (2002) A New Technique for Calculating Adomian Polynomials for Nonlinear Polynomials. Applied Mathematics and Computation, 111, 33-51. https://doi.org/10.1016/S0096-3003(99)00063-6

[11] Wazwaz, A.M. (2002) Partial Differential Equations Methods and Applications. Netherland Balkema Publisher.

[12] Rèpaci, A. (1990) Nonlinear Dynamical Systems: On the Accuracy of Adomian's Decomposition Method. Applied Mathematics Letters, 3, 35-39. https://doi.org/10.1016/0893-9659(90)90042-A

[13] Khuri, S.A. (2001) A Laplace Decomposition Algorithm Applied to Class of Nonlinear Differential Equations. Journal of Applied Mathematics, 4, 141-155. https://doi.org/10.1155/S1110757X01000183

[14] Khuri, S.A. (2004) A New Approach to Bratu's Problem. Applied Mathematics and Computation, 147, 131-136. https://doi.org/10.1016/S0096-3003(02)00656-2

[15] Yusufoglu (Agadjanov), E. (2006) Numerical Solution of Duffing Equation by the Laplace Decomposition Algorithm. Applied Mathematics and Computation, 177, 572-580. https://doi.org/10.1016/j.amc.2005.07.072

[16] Cherruault, Y. and Adomian, G. (1993) Decomposition Methods: A New Proof of Convergence. Mathematical and Computer Modelling, 18, 103-106. https://doi.org/10.1016/0895-7177(93)90233-O 